\title{
Editorial
}

\section{Contribution of the International Archives of Otorhinolaryngology to COVID-19 Publications: A Bibliometric Analysis}

\author{
Adilson Marcos Montefusco ${ }^{10}$ Geraldo Pereira Jotz ${ }^{20}$ \\ ${ }^{1}$ Department of Otorhinolaryngology, University of São Paulo, São \\ Paulo, SP, Brazil \\ ${ }^{2}$ Department of Morphological Sciences, Federal University of Rio \\ Grande do Sul, Porto Alegre, RS, Brazil
}

Int Arch Otorhinolaryngol 2021;25(3):e329-e333.

COVID-19 is an emerging, rapidly evolving situation, and scientists all around the world are focused on finding and developing treatments for this infection. Their efforts have led to a number of scientific publications since the start of the coronavirus outbreak, which was declared as a pandemic by the World Health Organization; 147,535 scientific articles have been published (as indexed in PubMed on June 22, 2020). This was considered by Science as "one of the greatest explosions of scientific literature of all time." 1 In part, this event reflects editors' efforts to reduce the peer review time and increase the speed of publications, without affecting the quality of research. ${ }^{1,2}$

This article aims to analyze and map the articles regarding COVID-19 published in the journal International Archives of Otorhinolaryngology (IAO) from a bibliometric perspective.

\section{Methods}

Initially, we included 24 COVID-19 documents published in the journal IAO. For this purpose, we used the search terms “COVID” OR “Coronavirus" OR "SARS-CoV-2" OR “2019nCoV" in the Scopus database and exported the data in CSV format: citation information, bibliographic information, and abstract \& keywords. Subsequently, we included four more documents manually, which were still in the publication phase, totaling 28 documents. All data were retrieved on June 22, 2020.

We used the VosViewer software (version 1.6.16) to analyze country collaboration and the co-occurrence of keywords. We used Google Drive Word Cloud to check the most frequent words in the full text of the manuscripts. ${ }^{3,4}$

Address for correspondence Adilson Montefusco, MSc, Research Center, Department of Otorhinolaryngology, University of São Paulo, São Paulo, SP, Brazil. Rua Artur de Azevedo, 46, São Paulo, SP, 05404-000, Brazil

(e-mail: montefuscos@gmail.com).

\section{The Contribution of IAO to Publications on COVID-19}

In early 2020, the editor-in-chief of the IAO journal and the team pledged to rapidly follow the submissions of COVID-19 articles and publish them online shortly after acceptance and editorial production.

On April 13, 2020, the IAO published its first articles on COVID-19; a total of 28 documents were published including nine $(32.14 \%)$ original research articles, seven (25\%) editorials, four (14.29\%) systematic reviews (including meta-analysis), four $(14.29 \%)$ letters to the editor, two (7.14\%) opinion articles, and two (7.14\%) update articles. The average period between submission and acceptance was 46 days and that between acceptance and online publication was 83 days ( $\mathbf{- T a b l e ~} \mathbf{1}$ ).

Of the 28 published articles, Brazil had the highest number of publications with six (45.71\%) documents followed by the United States with four (11.43\%) and India with three (8.57\%; - Fig. 1). To analyze international cooperation in terms of COVID-19 articles, i.e., when two or more countries participated in the same study, we built a collaboration network using the VosViewer software. Our dataset involved six countries; different colors indicate different groups, size of the circle represents the number of publications, and thickness of the line represents the strength of collaborations between the countries that it connects (-Fig. 2). - Figure 3 shows the 15 affiliations/institutions that had the highest participation in these studies.

To analyze the co-occurrence of keywords, we used VosViewer to view the most commonly used terms. All keywords (author + index keywords) that occurred more than twice were entered in the final analysis. The most frequent keywords were
DOI https://doi.org/ 10.1055/s-0041-1733778. ISSN 1809-9777.

\footnotetext{
(c) 2021. Fundação Otorrinolaringologia. All rights reserved. This is an open access article published by Thieme under the terms of the Creative Commons Attribution-NonDerivative-NonCommercial-License, permitting copying and reproduction so long as the original work is given appropriate credit. Contents may not be used for commercial purposes, or adapted, remixed, transformed or built upon. (https://creativecommons.org/ licenses/by-nc-nd/4.0/) Thieme Revinter Publicações Ltda., Rua do Matoso 170, Rio de Janeiro, RJ, CEP 20270-135, Brazil
} 


\begin{tabular}{|c|c|c|c|c|c|c|c|c|c|c|c|c|c|c|c|}
\hline 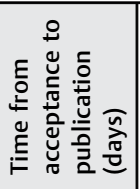 & $*$ & * & $\stackrel{\infty}{\sim}$ & * & * & $\stackrel{\infty}{\circ}$ & g & $\stackrel{\text { m }}{ }$ & * & 웅 & * & gి & $\bar{\infty}$ & à & * \\
\hline 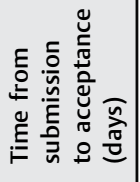 & $*$ & * & - & * & * & 0 & a & $m$ & * & r & * & o & 0 & $\approx$ & * \\
\hline 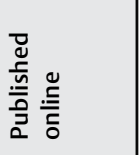 & $\frac{\stackrel{D}{0}}{\frac{d}{d}}$ & $\begin{array}{l}\stackrel{0}{\tilde{N}} \\
\frac{\stackrel{N}{m}}{m} \\
\frac{\vec{d}}{d}\end{array}$ & 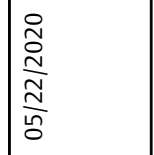 & $\begin{array}{l}\stackrel{0}{0} \\
\frac{d}{N} \\
\frac{1}{8}\end{array}$ & $\mid \begin{array}{l}\frac{2}{0} \\
\frac{d}{N} \\
\frac{N}{8}\end{array}$ & $\mid \begin{array}{l}\stackrel{0}{0} \\
\stackrel{0}{1} \\
\frac{m}{\frac{m}{0}}\end{array}$ & 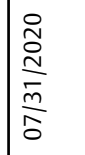 & 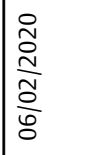 & 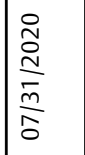 & 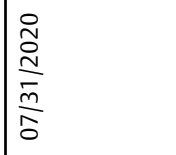 & 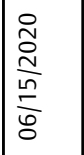 & $\begin{array}{l}\stackrel{0}{0} \\
\frac{1}{2} \\
\frac{a}{8} \\
\frac{0}{8}\end{array}$ & 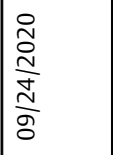 & $\mid \begin{array}{l}\stackrel{2}{0} \\
\stackrel{D}{0} \\
\frac{0}{0} \\
\frac{m}{8}\end{array}$ & 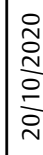 \\
\hline 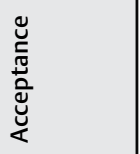 & * & * & 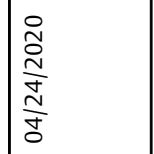 & * & * & 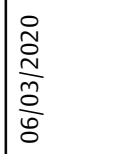 & 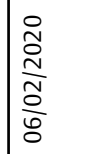 & 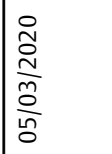 & * & 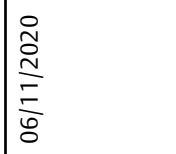 & * & 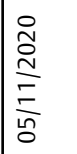 & 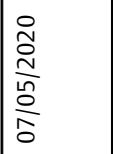 & 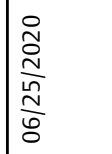 & * \\
\hline $\begin{array}{l}\text { co } \\
\underline{\underline{\underline{\omega}}} \\
\underline{\underline{E}}\end{array}$ & * & * & 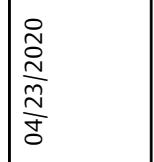 & * & * & 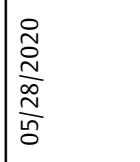 & 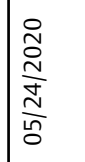 & 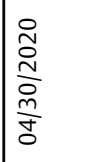 & 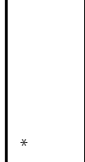 & $\begin{array}{l}\frac{8}{8} \\
\frac{1}{0} \\
\frac{1}{2} \\
\frac{0}{2}\end{array}$ & * & 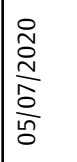 & 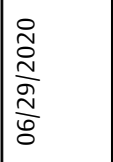 & $\begin{array}{l}\frac{0}{2} \\
\frac{a}{1} \\
\frac{0}{0} \\
\frac{0}{8}\end{array}$ & * \\
\hline 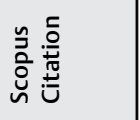 & $\stackrel{\stackrel{\sim}{\sim}}{\sim}$ & $\circ$ & in & - & - & - & - & - & - & 0 & 0 & 0 & $m$ & - & 0 \\
\hline $\begin{array}{l}\vec{E} \\
\text { 产 }\end{array}$ & 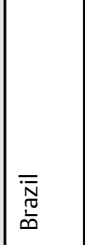 & \begin{tabular}{|l|l}
$\overline{\bar{N}}$ \\
$\bar{N}$
\end{tabular} & $\mid \begin{array}{c}\overline{\overline{\mathbb{N}}} \\
\bar{N}\end{array}$ & 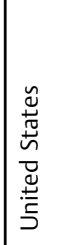 & 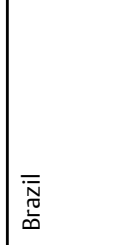 & 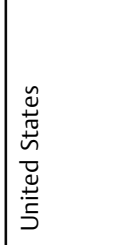 & 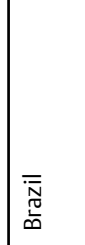 & 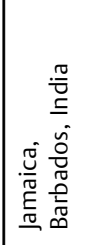 & $\begin{array}{l}\text { 䓂 } \\
\underline{\underline{\underline{I}}}\end{array}$ & 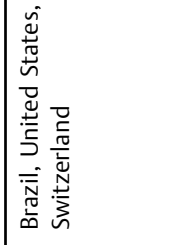 & 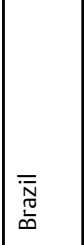 & $\frac{\lambda}{ \pm}$ & 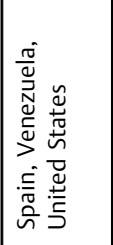 & 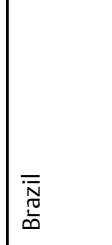 & \begin{tabular}{|l}
$\overline{\bar{N}}$ \\
$\bar{N}$
\end{tabular} \\
\hline$\stackrel{\mathscr{D}}{\underline{\underline{E}}}$ & 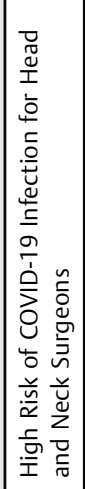 & 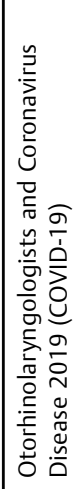 & 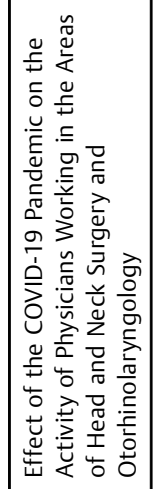 & 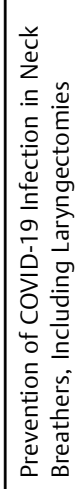 & 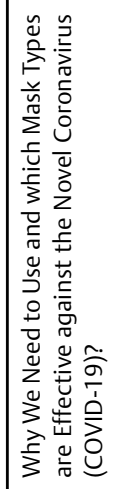 & 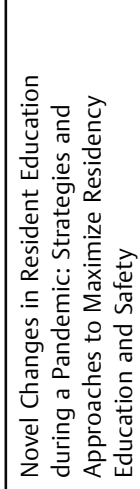 & 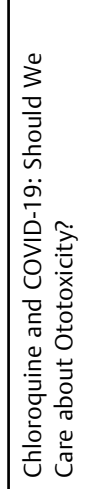 & 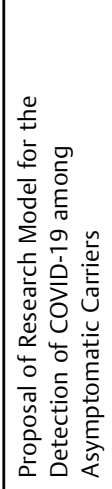 & 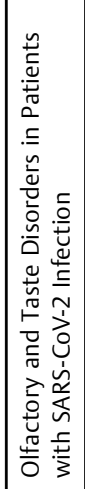 & 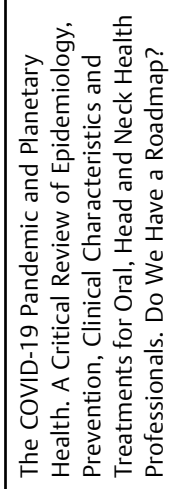 & 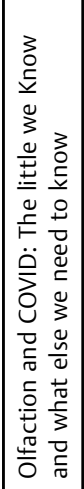 & 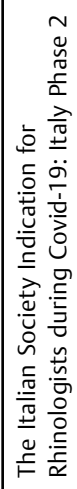 & 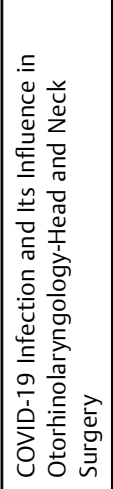 & 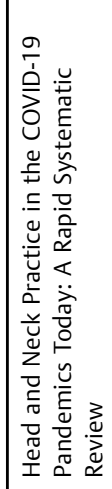 & \\
\hline$\stackrel{\Perp}{\stackrel{2}{2}}$ & 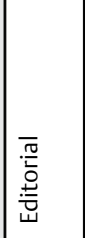 & 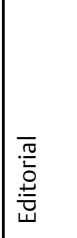 & 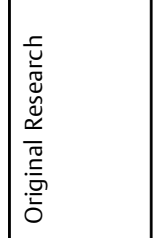 & 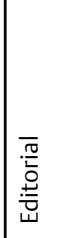 & 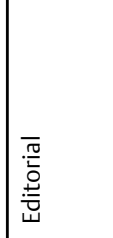 & 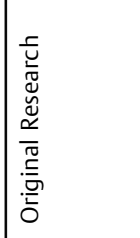 & 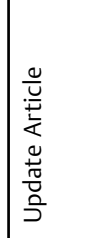 & 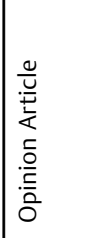 & 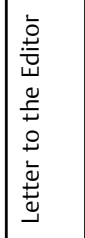 & 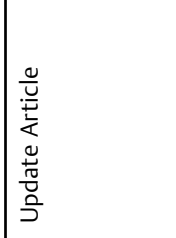 & 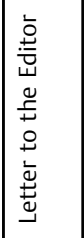 & 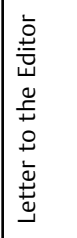 & 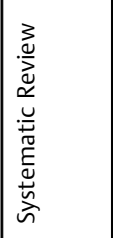 & 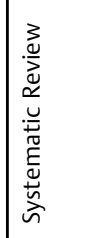 & 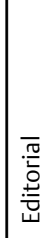 \\
\hline 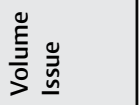 & 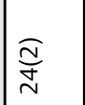 & 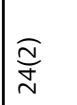 & 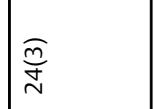 & 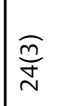 & 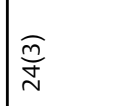 & 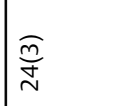 & $\widehat{\stackrel{m}{d}}$ & 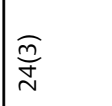 & $\widehat{\widehat{m}}$ & $\underset{\widetilde{N}}{\stackrel{\tilde{m}}{d}}$ & $\underset{\substack{\tilde{J} \\
\sim}}{ }$ & $\widehat{\widehat{N}} \underset{\sim}{\stackrel{N}{N}}$ & $\underset{\sim}{\stackrel{\Im}{J}}$ & 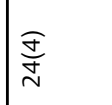 & $\underset{\sim}{\stackrel{7}{7}}$ \\
\hline 离 & 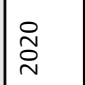 & 空 & & 灾 & 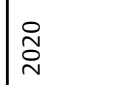 & ્ָ & 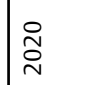 & 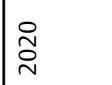 & 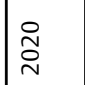 & 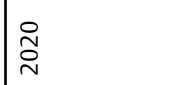 & 空 & 空 & 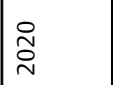 & 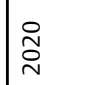 & స్ \\
\hline
\end{tabular}




\begin{tabular}{|c|c|c|c|c|c|c|c|c|c|c|c|c|c|c|c|}
\hline 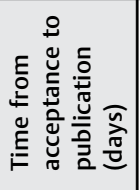 & & $\hat{\theta}$ & $\underline{\Sigma}$ & $\curvearrowright$ & $\infty$ & * & * & $\stackrel{\infty}{\circ}$ & $\stackrel{\Sigma}{\Sigma}$ & $\underset{\sigma}{\sigma}$ & * & * & * & * & $\begin{array}{l}\infty \\
\infty \\
\infty \\
\infty\end{array}$ \\
\hline 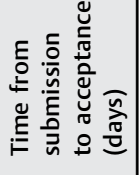 & & $\stackrel{m}{m}$ & $\bar{\sim}$ & ๓̊ & 望 & * & * & $\curvearrowright$ & $m$ & $\stackrel{\infty}{\wedge}$ & $\stackrel{\text { กี }}{\stackrel{2}{2}}$ & $\stackrel{\Xi}{=}$ & $\stackrel{m}{\wedge}$ & $\underset{\mathcal{Z}}{\mathcal{Z}}$ & $\begin{array}{l}\stackrel{n}{0} \\
\dot{g}\end{array}$ \\
\hline 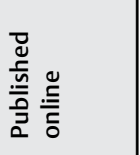 & & 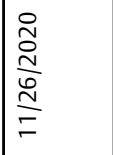 & 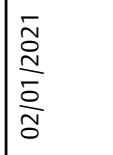 & 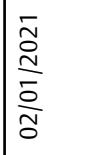 & $\frac{\bar{c}}{\stackrel{\bar{\alpha}}{c}}$ & 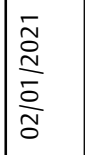 & 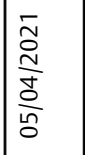 & $\begin{array}{l}\bar{̃} \\
\stackrel{N}{N} \\
\frac{\hat{N}}{\tilde{N}}\end{array}$ & $\mid \begin{array}{l}\bar{a} \\
\frac{a}{\sigma} \\
\frac{a}{\bar{d}}\end{array}$ & 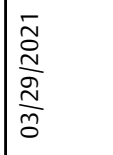 & * & $*$ & * & * & . \\
\hline 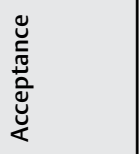 & & 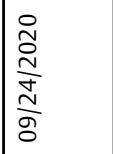 & $\mid \begin{array}{l}\stackrel{0}{0} \\
\stackrel{0}{0} \\
\frac{\infty}{\infty} \\
\stackrel{0}{\infty} \\
o\end{array}$ & $\begin{array}{l}\stackrel{0}{0} \\
\stackrel{0}{0} \\
\frac{0}{6} \\
\stackrel{1}{1}\end{array}$ & 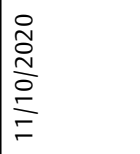 & * & * & 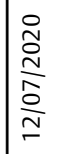 & 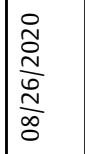 & 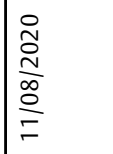 & 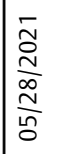 & $\mid \begin{array}{l}\bar{a} \\
\frac{a}{1} \\
\frac{a}{a} \\
\frac{a}{0}\end{array}$ & 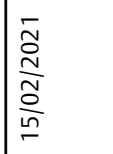 & 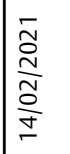 & . \\
\hline 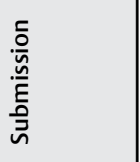 & & 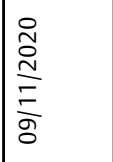 & 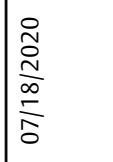 & $\begin{array}{l}\stackrel{0}{\mathcal{N}} \\
\frac{\hat{D}}{\infty} \\
\frac{0}{8} \\
\frac{0}{0}\end{array}$ & $\begin{array}{l}\stackrel{0}{1} \\
\stackrel{0}{1} \\
\frac{0}{0} \\
\frac{0}{0}\end{array}$ & * & * & $\mid \begin{array}{l}\frac{2}{a} \\
\frac{a}{\frac{1}{8}} \\
\frac{8}{8}\end{array}$ & 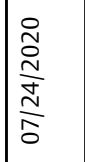 & 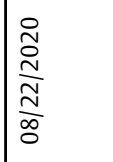 & 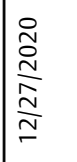 & $\begin{array}{l}\stackrel{0}{2} \\
\stackrel{2}{\frac{1}{2}} \\
\frac{2}{\ln }\end{array}$ & 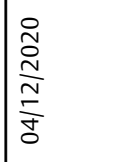 & 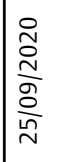 & . \\
\hline 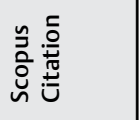 & & ڤn & $\sim$ & $\sim$ & - & 0 & 0 & 0 & 0 & 0 & 苂 & * & * & * & $\stackrel{n}{N}$ \\
\hline 訔 & & 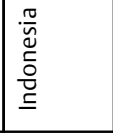 & \begin{tabular}{|l} 
ते \\
\pm
\end{tabular} & 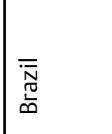 & 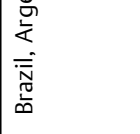 & 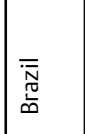 & 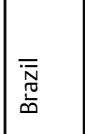 & 窵 & 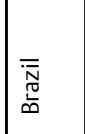 & 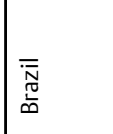 & \begin{tabular}{|l} 
离 \\
$\underline{\underline{\underline{C}}}$
\end{tabular} & 蓄 & 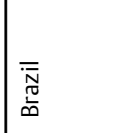 & 蓄 & . \\
\hline$\stackrel{\varrho}{\stackrel{\varrho}{E}}$ & 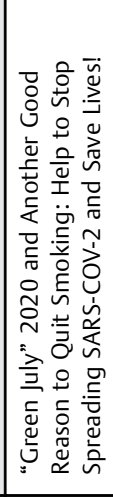 & 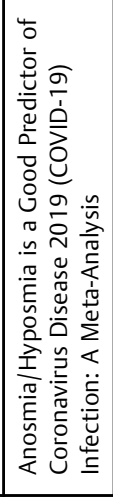 & 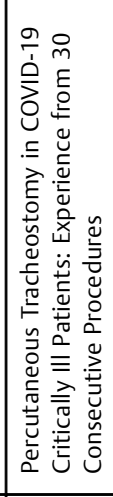 & 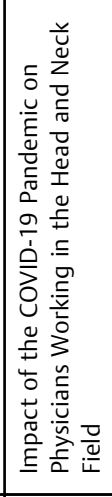 & 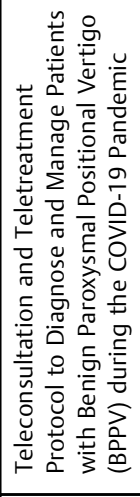 & 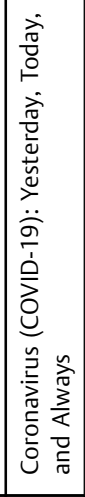 & 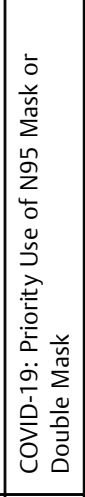 & 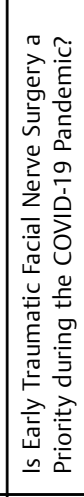 & 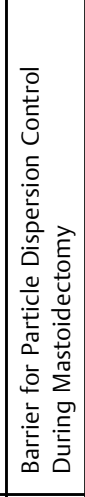 & 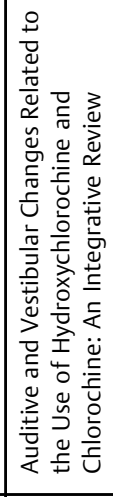 & 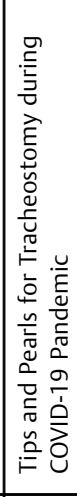 & 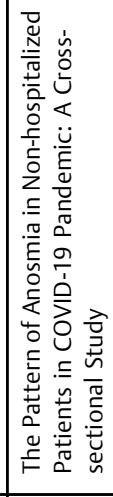 & 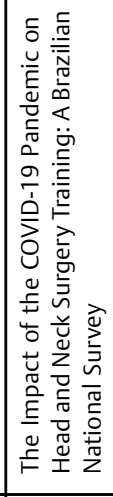 & 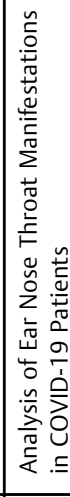 & ' \\
\hline 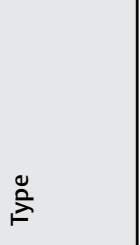 & & 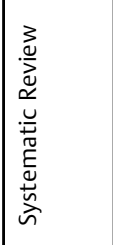 & 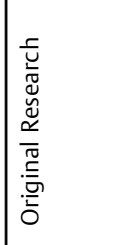 & 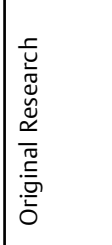 & 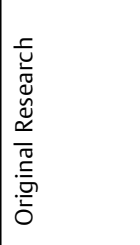 & 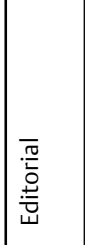 & 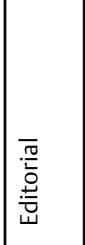 & 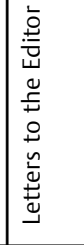 & 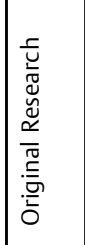 & 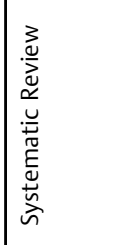 & 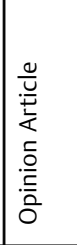 & 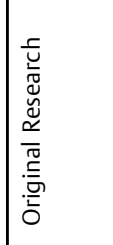 & 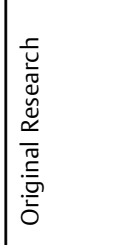 & 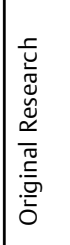 & . \\
\hline 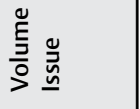 & & 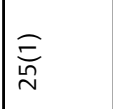 & $\underset{\text { స్ }}{\stackrel{్}{\sim}}$ & 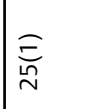 & 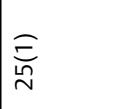 & 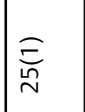 & 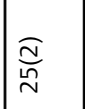 & $\underset{\underset{N}{N}}{\stackrel{\widehat{N}}{N}}$ & 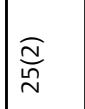 & 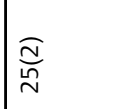 & 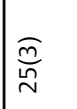 & $\widehat{\widetilde{N}}$ & $\widehat{\stackrel{\tilde{n}}{N}}$ & $\widehat{\widehat{N}}$ & . \\
\hline 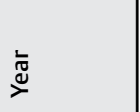 & & $\overline{\tilde{N}}$ & $\overline{\tilde{N}}$ & 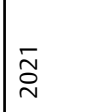 & $\overline{\widetilde{N}}$ & $\overline{\widetilde{N}}$ & 六 & 六 & $\overline{\tilde{N}}$ & 衣 & $\overline{\widetilde{\sim}}$ & $\overline{\tilde{N}}$ & 衣 & $\overline{\widetilde{N}}$ & 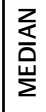 \\
\hline
\end{tabular}




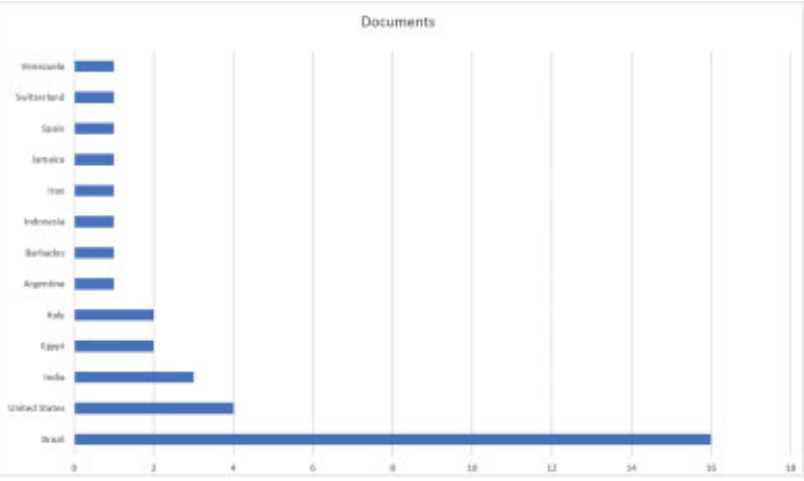

Fig. 1 Documents by country or territory

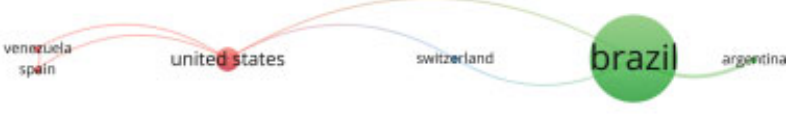

A vosviewer

Fig. 2 Visualization of the country collaboration network

“coronavirus disease 2019" (total link strength: 313), "pandemic" (total link strength: 189), "COVID-19" (total link strength: 141), and "aerosol" (total link strength: 123) (- Fig. 4).

In addition, we created a full-text word cloud (title, abstract, keywords, and manuscript body) to show the most frequently used words in the manuscripts. The most frequent terms were "COVID" $(n=668)$, "patients" $(n=645)$, and "pandemic" ( $\mathrm{n}=204$; - Fig. 5).

For the citation metrics, we considered 24 documents (published in the editions from April 2020 to 2021) and excluded four documents published in this edition (July 2021) because they did not allow sufficient time to accumulate citations. The 24 documents, according to the Scopus database (on June 22, 2021), received a total of 60 citations (average 2.5 citations per article) (-Table 1). The average number of citations of COVID-19 articles was higher than the average number of citations received by the IAO journal in 2020, citations per document 2 years $=1.191$ (according to the Scimago database) ${ }^{5}$ This value indicates the number of citations received by the documents of a journal divided by the total number of documents published in that journal.

\section{Final Comments}

In this study, we used descriptive bibliometric analysis to understand the profile of authors and the topics and to monitor the evolution of citations. "Hot" and urgent topics like this one usually lead to a significant increase in the number of publications and citations; therefore, these documents require monitoring, and bibliometric analysis provides a guide for the important topics and trends for future research.

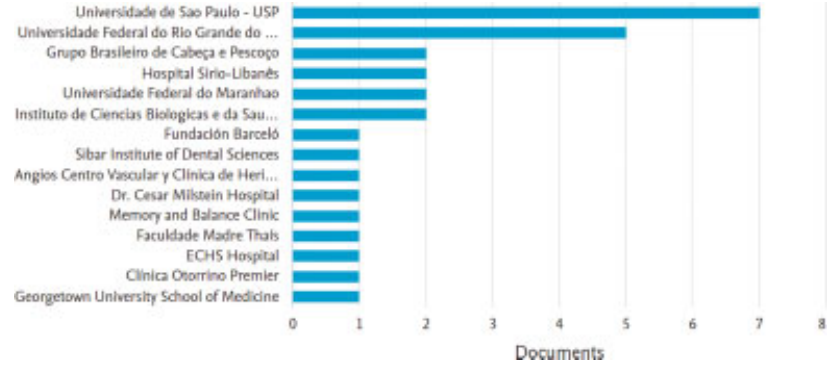

Fig. 3 Documents by affiliation; Source: Scopus

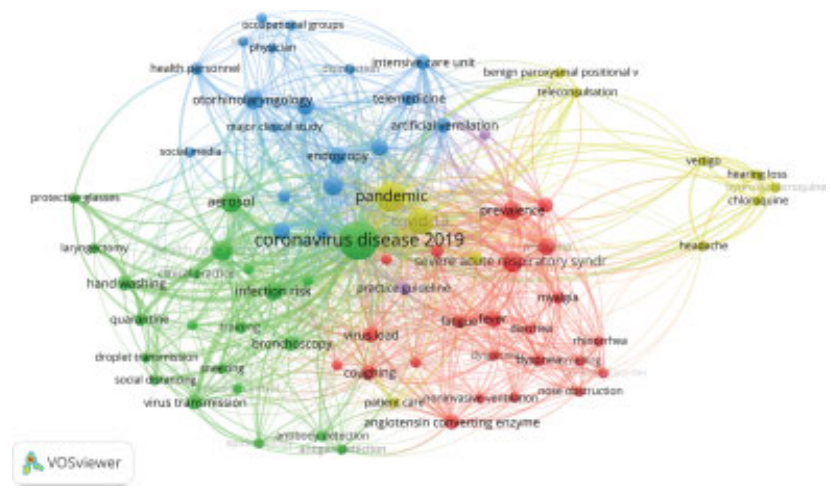

Fig. 4 Keyword co-occurrence analysis by VosViewer

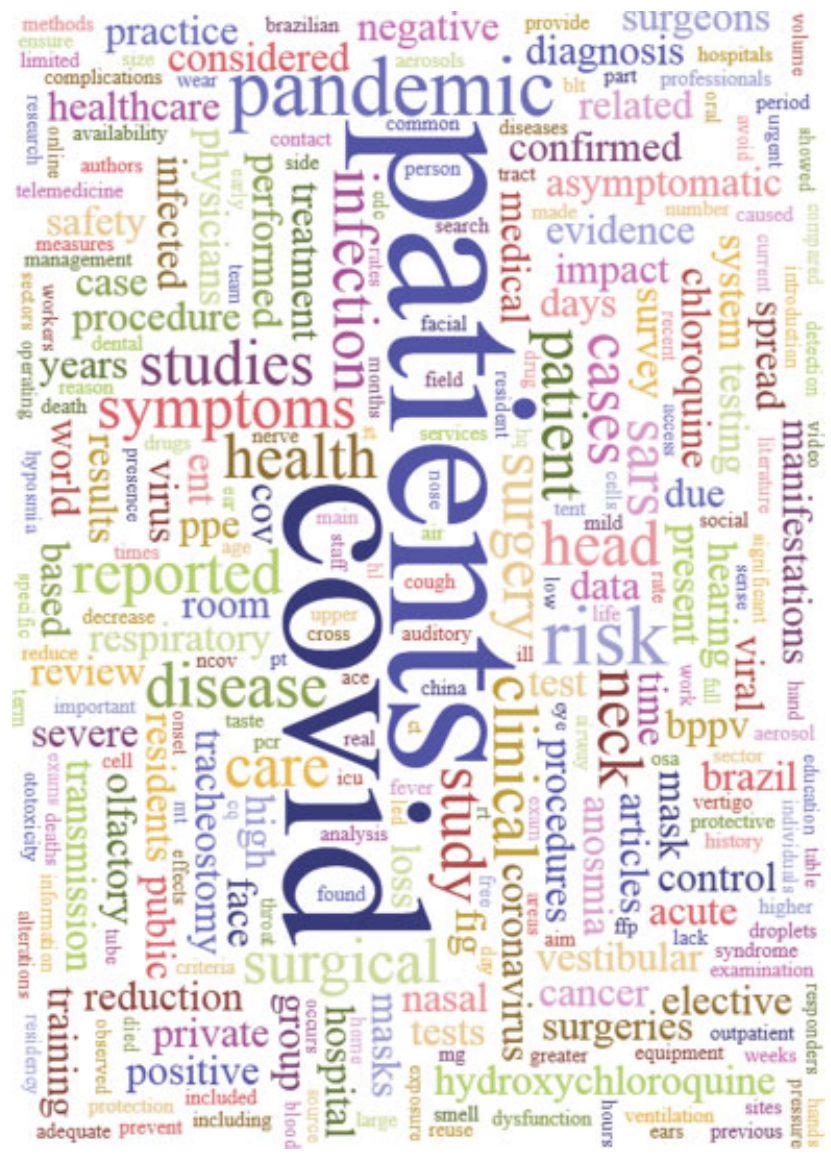

Fig. 5 Word cloud created on Google Drive. The font size represents the frequency of occurrence. 


\section{Conflict of Interest}

The authors have no conflict of interests to declare.

\section{Acknowledgements}

We/The authors thank Crimson Interactive Pvt. Ltd. (Ulatus) - www.ulatus.com.br for their assistance in manuscript translation and editing.

\section{References}

1 Brainard J. Scientists are drowning in COVID-19 papers. Can new tools keep them afloat? [Internet]. Science 2020. Accessed June 22, 2020 at: https://doi.org/10.1126/science.abc7839
2 Fassin Y. Research on Covid-19: a disruptive phenomenon for bibliometrics. Scientometrics 2021;126(06):5305-5319. Doi: 10.1007/s11192-021-03989-w

3 Yu Y, Li Y, Zhang Z, et al. A bibliometric analysis using VOSviewer of publications on COVID-19. Ann Transl Med 2020;8(13):816. Doi: $10.21037 /$ atm-20-4235

$4 \mathrm{Wu}$ W, Xie Y, Liu X, et al. Analysis of Scientific Collaboration Networks among Authors, Institutions, and Countries Studying Adolescent Myopia Prevention and Control: A Review Article. Iran J Public Health 2019;48(04):621-631

5 SCImago. SJR - SCImago Journal \& Country Rank [Internet]. 2020 [about International Archives of Otorhinolaryngology]. Accessed June 22, 2020 at: https://www.scimagojr.com/journalsearch. php?q=18099777 